\title{
Perfil nutricional e benefícios do azeite de abacate (Persea americana): uma revisão integrativa
}

\author{
Nutritional profile and benefits of avocado oil (Persea americana): an integrative review
}

\section{Carlos Alberto Nogueira-de-Almeida ${ }^{1 *}$, Fábio da Veiga Ued², Carla Cristina João Nogueira de Almeida ${ }^{3}$, Ane Cristina Fayão Almeida², Luiz Antonio Del Ciampo², Ivan Savioli Ferraz², Luiz Fernando de Oliveira da Silva Carolina Ruiz Zambom ${ }^{4}$, Adelson Francisco de Oliveira ${ }^{5}$}

\author{
1 Universidade Federal de São Carlos (UFSCar), Departamento de Medicina, São Carlos/SP - Brasil \\ ${ }^{2}$ Universidade de São Paulo (USP), Faculdade de Medicina de Ribeirão Preto, Departamento de Puericultura e Pediatria, Ribeirão Preto/SP - Brasil \\ ${ }^{3}$ Clínica Nutre Serviços de Consultoria e Assessoria, Ribeirão Preto/SP - Brasil \\ ${ }^{4}$ Empresa de Pesquisa Agropecuária de Minas Gerais (EPAMIG), Campo Experimental, Maria da Fé/MG - Brasil \\ ${ }^{5}$ Empresa de Pesquisa Agropecuária de Minas Gerais (EPAMIG), Centro Tecnológico do Sul de Minas, Universidade Federal de Lavras (UFLA), \\ Lavras/MG - Brasil
}

\section{${ }^{*}$ Corresponding Author}

Carlos Alberto Nogueira-de-Almeida, Universidade Federal de São Carlos (UFSCar), Departamento de Medicina, UFSCAR, Rodovia Washington Luiz, km 235, CEP: 13565-905, São Carlos/SP - Brasil, e-mail: dr.nogueira@me.com

Cite as: Nutritional profile and benefits of avocado oil (Persea americana): an integrative review. Braz. J. Food Technol., v. 21, e2017214, 2018.

Received: Nov. 24, 2017; Accepted: May 30, 2018

\section{Resumo}

O objetivo do presente estudo foi realizar uma revisão integrativa sobre a qualidade do perfil lipídico e dos compostos bioativos presentes no azeite de abacate, bem como sobre os seus potenciais benefícios para a saúde humana. Foram identificados trabalhos publicados nas bases de dados Scopus, PubMed, Lilacs, SciELO e Web of Science, além de Biblioteca Digital de Teses e Dissertações. A pesquisa encontrou dados referentes ao perfil lipídico e ao teor de substâncias bioativas presentes no azeite de abacate extraído das variedades Margarida, Quintal, Fortuna, Ouro Verde, Hass e Fuerte. O azeite de abacate caracteriza-se por apresentar, em média, teores elevados de ácidos graxos monoinsaturados (60\%), teor intermediário de ácidos graxos saturados (24\%) e baixo teor de ácidos graxos poli-insaturados (16\%). O ácido graxo oleico (ômega-9) é o de maior concentração (aproximadamente 55\%). Os principais esteróis, tocoferóis e carotenoides encontrados foram $\beta$-sitosterol, $\alpha$-tocoferol e luteína, respectivamente. O azeite de abacate possui propriedades físico-químicas semelhantes às do azeite de oliva. Em modelos animais, é capaz de prevenir a disfunção mitocondrial cerebral e hepática induzida pelo diabetes, associado à diminuição de estresse oxidativo, LDL-c e peroxidação lipídica. Seus benefícios são atribuídos principalmente às concentrações de ácido oleico, por sua atividade cardioprotetora, ao $\beta$-sitosterol, por sua propriedade anti-inflamatória, e ao $\alpha$-tocoferol e à luteína, pela propriedade antioxidante. Assim, o azeite de abacate se destaca pelo teor elevado de ácidos graxos monoinsaturados e de compostos bioativos, como tocoferóis, carotenoides e fitoesteróis. Análises em modelos animais, aliadas às análises de qualidade e segurança, atestam os benefícios e a possibilidade de introdução do referido azeite para uso comestível, substituindo óleos de qualidade inferior e criando nova alternativa gastronômica ao consumidor.

Palavras-chave: Persea; Óleos; Óleos vegetais; Composição de alimentos; Saúde; Alimentação.

\section{Abstract}

The aim of this study was to carry out an integrative review on the quality of the lipid profile and bioactive compounds present in avocado oil, as well as its potential benefits to human health. Papers were identified, published in the Scopus, PubMed, Lilacs, SciELO and Web of Science databases, as well as in the Digital Thesis and Dissertation Library. The survey found data on the lipid profiles and bioactive contents of avocado oils extracted from the varieties Margarida, Quintal, Fortuna, Ouro Verde, Hass and Fuerte. Avocado oil is characterized by high levels of monounsaturated fatty acids (60\%), 
intermediate contents of saturated fatty acids (24\%) and low contents of polyunsaturated fatty acids (16\%). The fatty acid oleic acid (omega-9) is the one with the highest concentration (approximately 55\%). The major sterols, tocopherols and carotenoids found were $\beta$-sitosterol, $\alpha$-tocopherol and lutein, respectively. Avocado oil has physicochemical properties similar to those of olive oil. In animal models, it is able to prevent the cerebral and hepatic mitochondrial dysfunction induced by diabetes, associated with a reduction in oxidative stress, LDL-c and lipid peroxidation. Its benefits are attributed mainly to the oleic acid concentrations for their cardio-protective activity, $\beta$-sitosterol for its anti-inflammatory properties, and $\alpha$-tocopherol and lutein for their antioxidant properties. Thus avocado oil stands out for its high contents of monounsaturated fatty acids and bioactive compounds such as tocopherols, carotenoids and phytosterols. Analyses in animal models coupled with quality and safety analyses have attested to the benefits of avocado oil and the possibility of introducing it for edible use, replacing inferior quality oils and creating a new gastronomic alternative for the consumer.

Keywords: Persea; Oils; Plant oils; Food composition; Health; Feeding.

\section{Introdução}

O abacate (Persea americana Mill.) é um fruto pertencente ao gênero Persea da família Lauraceae, com cerca de 150 espécies, originário do continente americano, especialmente do México, da América Central e das Antilhas. Possui boa qualidade nutricional devido ao seu elevado conteúdo de lipídios, associado a proteínas, vitaminas e minerais; apresenta também grande potencial econômico devido ao aproveitamento de seus componentes na indústria farmacêutica, de cosméticos e de biocombustível (SOARES; ITO, 2000; MASSAFERA et al., 2010; QIN; ZHONG, 2016).

A produção mundial de abacate está em constante aumento, com crescimento de $19 \%$ no período de 2006 a 2012. O Brasil é o oitavo produtor mundial (159,9 mil toneladas em 2013) (PEREIRA, 2015), sendo o abacate a décima sétima fruta mais produzida no país (JORGE, 2014). A diversidade climática existente no Brasil torna o país um grande produtor do fruto, principalmente para o consumo in natura. A produção de abacates encontra-se distribuída por todo o território nacional, concentrando-se nos Estados de São Paulo e Minas Gerais (JORGE, 2014). Observe-se que o abacateiro é uma planta frutífera altamente produtiva por unidade de área cultivada (TANGO; TURATTI, 1992).

No Brasil, o abacate, culturalmente, é consumido na forma de sobremesas, com leite, açúcar, mel ou suco de limão. Em outros países, é consumido na forma de saladas, acompanhando cebola e queijo, além de sopas, molhos e conservas. Na área industrial, o abacate é utilizado para extração de azeite e etanol, visando à a geração de biocombustível e fabricação de tintas. Após a extração, o azeite também é utilizado por indústrias farmacêuticas e de cosméticos, devido ao alto teor de vitamina $E$ ( $\alpha$-tocoferol) em sua composição, o que lhe confere propriedades regenerativas e fácil absorção no tecido epidérmico (MASSAFERA et al., 2010).

Além disso, o azeite extraído também pode ser refinado e utilizado na culinária (GÓMEZ-LÓPEZ, 1999). A produção mundial de azeite de abacate que abastece o setor de alimentos é pequena e há amplo espaço a ser explorado no mercado. O abacate pode render até 2.800 litros de azeite por hectare contra o rendimento de 400 litros de azeite por hectare da soja, além de o abacateiro ser uma planta perene (PEREIRA, 2015). Ainda pouco consumido no Brasil, o azeite já é utilizado como tempero para saladas em regiões da Nova Zelândia, do Canadá, dos Estados Unidos e da Europa.

Sabe-se que o abacate possui elevado teor lipídico em sua polpa, cuja composição nutricional é capaz de auxiliar na prevenção de doenças crônicas não transmissíveis. Contudo, é necessário elucidar se o azeite extraído do fruto possui qualidades nutricionais para se tornar uma alternativa saudável de consumo para a população. Assim, o objetivo do presente estudo foi realizar uma revisão da literatura científica a respeito da qualidade do perfil lipídico e dos compostos bioativos presentes no azeite de abacate, bem como acerca dos seus potenciais benefícios para a saúde humana.

\section{Material e métodos}

Trata-se de uma revisão integrativa da literatura, realizada mediante o cumprimento das seguintes etapas: 1) elaboração da pergunta norteadora, definição dos descritores e dos critérios para inclusão/exclusão de artigos; 2) amostragem (seleção dos artigos); 3) categorização dos estudos; 4) definição das informações a serem extraídas dos trabalhos revisados; 5) análise e discussão a respeito das tecnologias usadas/desenvolvidas; 6) síntese do conhecimento evidenciado nos artigos analisados e apresentação da revisão integrativa (MENDES et al., 2008).

Estabeleceu-se a seguinte pergunta norteadora: "Qual o perfil nutricional do azeite de abacate e seus benefícios para a saúde humana?". Os critérios de inclusão adotados no presente estudo foram: a publicação ter como temática a análise da composição química e os benefícios à saúde do azeite de abacate; publicações classificadas como artigo original, revisões bibliográficas, livros, teses, dissertações ou resumos de anais de eventos científicos, com data limite de publicação até agosto de 2017 , divulgadas em língua inglesa, espanhola e portuguesa; 
Perfil nutricional e benefícios do azeite de abacate (Persea americana): uma revisão integrativa

Nogueira-de-Almeida, C. A. et al.

publicações completas com resumos disponíveis e indexados nas bases de dados: Scopus, PubMed, Lilacs, SciELO, Web of Science e Biblioteca Digital de Teses e Dissertações. Foram excluídos os editoriais, as cartas ao editor, os estudos reflexivos e relatos de caso, bem como estudos que não abordassem a temática relevante ao objetivo desta revisão.

A pesquisa nas bases de dados foi realizada entre os meses de maio e agosto de 2017, sendo utilizados os Descritores em Ciências da Saúde (DECS) e o Medical Subject Headings (Mesh). Foram empregados os operadores booleanos "and" e "or" e os respectivos descritores: Persea (Persea), óleos (oils) e saúde (health).

Os resultados das buscas foram rastreados por três pesquisadores qualificados, a partir dos títulos e dos resumos dos artigos. Após a identificação dos estudos relevantes, a publicação completa foi adquirida e revisada pelos três profissionais, a fim de se determinar a elegibilidade para a inclusão final. Ao total, selecionaram-se 46 estudos, com base nos critérios de inclusão estabelecidos, conforme ilustrado na Figura 1.

Em relação à nomenclatura referente ao óleo extraído do abacate, considerou-se a seguinte premissa: óleos são extraídos de sementes, como os óleos de soja, de milho, entre outros. Azeites são extraídos da polpa de frutos, como o caso da oliva e do abacate. Assim, convencionou-se, na presente revisão, a utilizar o nome "azeite de abacate".

\section{Resultados e discussão}

\subsection{Raças e variedades}

Existem três grupos ou raças que representam o abacate, sendo elas: Mexicana (Persea americana var. drymifolia); Antilhana ( $P$. americana var. americana), e Guatemalense ( $P$. nubigena var. guatemalensis). Cada raça engloba diferentes variedades de abacate. $\mathrm{Na}$ raça Mexicana, as principais variedades do fruto são as cultivares Duke e Ettinger. Na raça Antilhana, as variedades mais importantes são Pollock, Simmonds e Fucks. Já na raça Guatemalense, as principais variedades são as cultivares Prince, Wagner, Linda e Rincon (CAMPOS, 1985; KOLLER, 1992).

Há ainda as variedades híbridas resultantes dos cruzamentos naturais entre as diferentes raças, como, por exemplo, as variedades Breda, Fortuna, Geada, Margarida, Ouro Verde e Quintal (híbridos das raças Antilhana e Guatemalense), e Hass e Fuerte (híbridos das raças Mexicana e Guatemalense). As cultivares Hass e Fuerte também são denominadas de "avocado" (PEREIRA, 2015), sendo valorizadas internacionalmente pelo alto teor de lipídios, apesar de suas dimensões reduzidas (FRANCISCO; BAPTISTELLA, 2005). A Figura 2 demonstra as principais variedades de abacate comercializadas no Brasil.

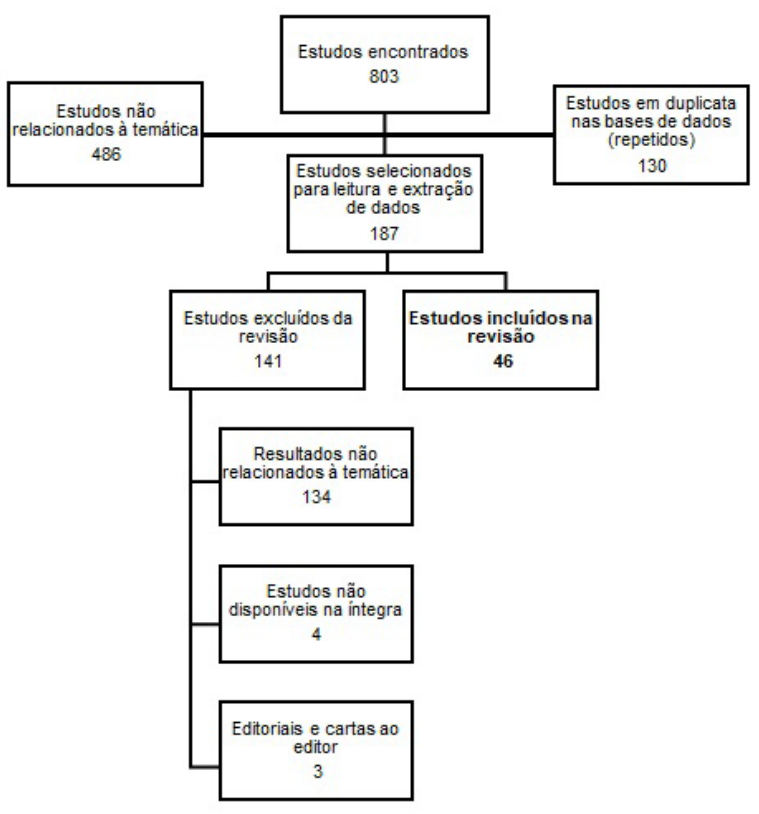

Figura 1. Processo de seleção dos artigos.

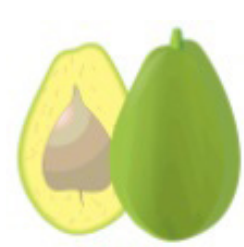

Fucks

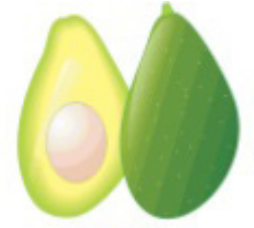

Geada

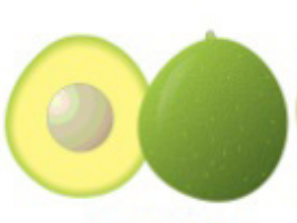

Margarida

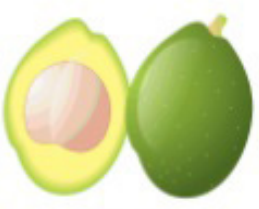

Breda

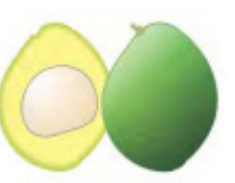

Ouro-Verde

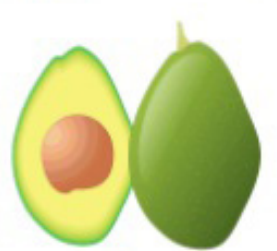

Fortuna

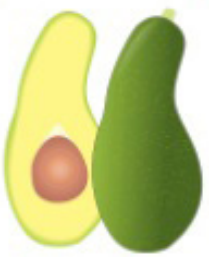

Quintal

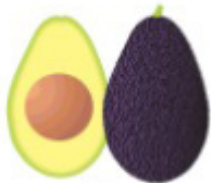

Hass

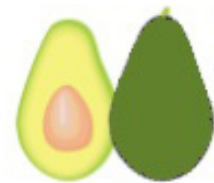

Fuerte

Figura 2. Principais variedades de abacate comercializadas no Brasil. Fonte: adaptado de Pereira (2015) e Hortiescolha (2017). 


\subsection{Perfil nutricional do abacate}

O abacate possui uma única semente (drupa) e é constituído por pericarpo (casca), mesocarpo (polpa) e endocarpo (semente). A fruta se caracteriza por possuir alto valor energético e nutricional, quando comparada a outros frutos tropicais. Sua utilidade na alimentação destaca-se, entre outros fatores, pela quantidade de ácidos graxos monoinsaturados presentes principalmente na polpa. A composição nutricional média da polpa do abacate está descrita na Tabela 1.

Além do teor elevado de lipídios, principalmente ácido oleico, pode-se observar um alto teor de proteínas,

Tabela 1. Composição nutricional média do abacate por 100 gramas de parte comestível.

\begin{tabular}{|c|c|}
\hline & Por $100 \mathrm{~g}$ de polpa \\
\hline Energia (kcal) & 96,0 \\
\hline Umidade (\%) & 83,8 \\
\hline Proteínas (g) & 1,2 \\
\hline Lipídios (g) & 8,4 \\
\hline Saturados (g) & 2,3 \\
\hline ácido mirístico [14:0] (g) & 0,01 \\
\hline ácido palmítico [16:0] (g) & 2,20 \\
\hline ácido esteárico [18:0] (g) & 0,10 \\
\hline ácido araquídico [20:0] (g) & 0,02 \\
\hline Monoinsaturados $(\mathrm{g})$ & 4,3 \\
\hline ácido palmitoleico [16:1 n-7] (g) & 0,20 \\
\hline ácido oleico [18:1 n-9] (g) & 4,12 \\
\hline ácido gadoleico [20:1] (g) & 0,02 \\
\hline Poli-insaturados (g) & 1,4 \\
\hline ácido linoleico [18:2 n-6] (g) & 1,29 \\
\hline ácido $\alpha$-linolênico [18:3 n-3] (g) & 0,08 \\
\hline Carboidratos $(\mathrm{g})$ & 6,0 \\
\hline Fibras (g) & 6,3 \\
\hline Cinzas (g) & 0,5 \\
\hline Cálcio (mg) & 8,0 \\
\hline Magnésio (mg) & 15,0 \\
\hline Manganês (mg) & 0,17 \\
\hline Fósforo (mg) & 22,0 \\
\hline Ferro (mg) & 0,2 \\
\hline Sódio (mg) & Traços \\
\hline Potássio (mg) & 206,0 \\
\hline Cobre (mg) & 0,15 \\
\hline Zinco (mg) & 0,2 \\
\hline Tiamina (mg) & Traços \\
\hline Riboflavina (mg) & 0,04 \\
\hline Piridoxina (mg) & Traços \\
\hline Niacina (mg) & Traços \\
\hline Vitamina A $(\mu \mathrm{g})$ & 7,04 \\
\hline Vitamina C (mg) & 8,7 \\
\hline Vitamina E (mg) & 2,66 \\
\hline
\end{tabular}

Fonte: IBGE (2011) e NEPA (2011). bem como de fibras, enquanto o conteúdo de açúcares é baixo em relação a outros frutos. A quantidade de cinzas indica uma boa constituição mineral da polpa. Contudo, deve-se ressaltar que a composição química da polpa do fruto pode variar de acordo com a época da colheita, o tempo de amadurecimento, a localidade das culturas e os fatores sazonais, como clima e umidade, além das diferenças próprias entre as variedades (SALGADO et al., 2008; QIN; ZHONG, 2016).

Apesar de haver alterações no perfil nutricional entre as principais variedades de abacate, poucos estudos realizaram comparações ou descreveram a composição nutricional de cada cultivar em específico. Essas informações são úteis para a análise e a seleção das variedades com o melhor perfil nutricional. Há pouco interesse na análise do teor de carboidratos e proteínas, devido à qualidade do fruto quanto à sua composição lipídica. A descrição do perfil nutricional de algumas variedades de abacate está apresentada na Tabela 2.

Observa-se que a polpa possui composição química bastante variável entre as diferentes espécies de abacate. O teor de gordura pode variar de 5\% a 30\%, aproximadamente. Note-se que, em uma mesma espécie, também pode haver diferenças quanto à concentração de azeite na polpa. A variedade Margarida, por exemplo, apresenta variação de 8,4\% a 26,3\%. É importante ressaltar que essas pesquisas realizaram as análises em frutos maduros e com metodologias similares para determinação da composição centesimal. Contudo, alguns estudos não informaram detalhadamente a época da colheita, o tempo de amadurecimento, a localidade das culturas e o clima e a umidade da região onde o fruto foi colhido. Estes fatores podem influenciar o perfil nutricional e o teor de umidade da polpa. Os frutos com baixo teor de umidade apresentarão maior conteúdo lipídico.

Apesar das variações na composição centesimal, as variedades Hass e Fuerte são aquelas que apresentam maiores concentrações de lipídios na polpa, acima de 20\% (TANGO et al., 2004; OLIVEIRA, 2011). Frutos com altos teores de gordura constituem-se em matéria-prima importante para obtenção de azeite (TANGO et al., 2004). Quanto ao teor de umidade, o avocado possui menor quantidade de água (abaixo de 70\%) em comparação às outras variedades, o que lhe confere maior concentração de nutrientes. O alto teor de umidade é o principal obstáculo para obtenção do azeite da polpa, por afetar o rendimento da extração (MONTANO et al., 1962).

Assim, analisando a composição nutricional dos frutos, principalmente o teor lipídico e a umidade, é possível constatar que o avocado (variedades Hass e Fuerte) apresenta maior potencial para extração de azeite. Entretanto, o avocado apresenta um custo elevado por litro de óleo extraído, fator que pode inviabilizar a extração do 
Perfil nutricional e benefícios do azeite de abacate (Persea americana): uma revisão integrativa

Nogueira-de-Almeida, C. A. et al.

Tabela 2. Perfil nutricional da polpa das variedades Margarida, Breda, Quintal, Fortuna, Ouro Verde, Hass e Fuerte.

\begin{tabular}{|c|c|c|c|c|c|c|c|}
\hline \multirow{2}{*}{ Variedade } & \multicolumn{6}{|c|}{ Composição centesimal (valor em \%) } & \multirow{2}{*}{ Referência } \\
\hline & Umidade & Carboidrato & Fibra & Proteína & Lipídio & Cinza & \\
\hline \multirow{4}{*}{ Margarida } & 58,3 & 7,25 & 4,85 & 2,54 & 26,3 & 0,6 & (SALGADO et al., 2008) \\
\hline & 81,2 & - & 2,95 & 0,74 & 8,7 & 0,8 & (OLIVEIRA et al., 2013) \\
\hline & 82,9 & - & - & 1,22 & 8,4 & 0,7 & (JORGE, 2014) \\
\hline & 79,1 & - & - & 1,19 & 14,0 & 0,8 & (ZÜGE, 2015) \\
\hline Breda & 78,2 & 6,37 & 1,55 & 1,73 & 15,8 & 0,6 & (GOUVEIA et al., 2015) \\
\hline \multirow{2}{*}{ Quintal } & 75,2 & - & 4,95 & 1,14 & 10,9 & 0,8 & (OLIVEIRA et al., 2013) \\
\hline & 77,4 & - & - & - & 14,7 & - & (TANGO et al., 2004) \\
\hline \multirow{4}{*}{ Fortuna } & 81,4 & - & 4,24 & 1,12 & 6,4 & 1,5 & (OLIVEIRA et al., 2013) \\
\hline & 87,2 & - & - & - & 5,9 & - & (TANGO et al., 2004) \\
\hline & 72,2 & - & 1,60 & 1,70 & 16,2 & 0,6 & (GALVÃO et al., 2014) \\
\hline & 76,9 & - & & 0,97 & 13,9 & 0,9 & (ZÜGE, 2015) \\
\hline \multirow{2}{*}{ Ouro Verde } & 78,9 & - & 4,13 & 0,86 & 8,4 & 0,9 & (OLIVEIRA et al., 2013) \\
\hline & 70,4 & - & - & - & 19,9 & - & (TANGO et al., 2004) \\
\hline \multirow{7}{*}{ Hass } & 70,1 & 1,17 & 1,62 & 0,24 & 25,2 & 1,6 & (DAIUTO et al., 2010) \\
\hline & 66,3 & 5,35 & - & 1,63 & 25,5 & 1,3 & (FERRARI, 2015) \\
\hline & 69,8 & - & - & 1,73 & 19,7 & 1,1 & (JORGE, 2014) \\
\hline & 62,1 & - & 7,14 & 1,90 & 21,1 & 2,4 & (OLIVEIRA et al., 2013) \\
\hline & 70,8 & - & - & 1,82 & 43,5 & 1,8 & (VINHA et al., 2013) \\
\hline & 57,2 & - & - & - & 31,1 & - & (TANGO et al., 2004) \\
\hline & 67,2 & - & - & 2,36 & 22,4 & 1,4 & (ZÜGE, 2015) \\
\hline \multirow{3}{*}{ Fuerte } & 76,2 & 5,65 & - & 1,47 & 15,1 & 1,6 & (FERRARI, 2015) \\
\hline & 60,9 & - & 4,72 & 1,88 & 26,1 & 1,3 & (OLIVEIRA et al., 2013) \\
\hline & 60,5 & - & - & - & 30,3 & - & (TANGO et al., 2004) \\
\hline
\end{tabular}

azeite desta variedade pela indústria brasileira e favorecer a extração advinda das demais variedades (ZÜGE, 2015).

\subsection{Perfil nutricional do azeite de abacate}

O óleo pode ser extraído tanto do mesocarpo quanto da semente, sendo maior a quantidade de azeite concentrada no mesocarpo (MASSAFERA et al., 2010). O azeite extraído da polpa apresenta maior concentração do ácido graxo oleico (MASSAFERA et al., 2010; GALVÃO et al., 2014), enquanto o óleo extraído da semente possui maior concentração dos ácidos graxos linoleico e $\alpha$-linolênico, além de conter agentes hepato-tóxicos (QIN; ZHONG, 2016). Para extração do azeite, o processo deve ser realizado quando os frutos estão maduros, isto é, com consistência amolecida, período no qual apresentam teores mais elevados de gordura na polpa (CAMPOS, 2009).

O azeite de abacate pode ser obtido por extração com solventes, por prensagem hidráulica (extração a frio), por centrifugação da polpa úmida, processos enzimáticos ou mesmo por processos convencionais de extração de óleo para sementes oleaginosas (TANGO et al., 2004). Dependendo do método de extração, o rendimento pode variar entre 56\% e 95\% de óleo extraído (TURATTI et al., 1985; TANGO; TURATTI, 1992; REDDY et al., 2012).
Além do rendimento após a extração, há o interesse de produtores por técnicas capazes de produzir azeites com alto teor de compostos bioativos, preservando as propriedades e o valor nutricional após a extração. A extração com solventes (éter de petróleo, hexano, acetona, entre outros) é o método mais comumente utilizado para extração de óleos, porém, apresenta algumas desvantagens, como a poluição ambiental e a permanência de resíduos de solventes nos produtos finais, o que limita a utilização do azeite em preparações alimentícias (QIN; ZHONG, 2016).

A extração por centrifugação da polpa úmida é um procedimento simples e de baixo custo, quando comparada aos demais métodos. Além disso, resulta em um produto de maior qualidade nutricional e pureza (QIN; ZHONG, 2016). Este método parece preservar um maior teor de esteróis e ácidos graxos ômega-3 no azeite (JORGE, 2014). Já a prensagem a frio é um processo rápido e extrai menor quantidade de azeite em comparação à extração com solventes. Entretanto, produz um azeite com maiores concentrações de antioxidantes (tocoferóis) e outros compostos bioativos, como o esqualeno (SANTOS et al., 2014).

A combinação entre diferentes processos de extração com o pré-tratamento (mecânico, enzimático ou térmico) do fruto pode resultar na obtenção de um azeite com 
Perfil nutricional e benefícios do azeite de abacate (Persea americana): uma revisão integrativa

Nogueira-de-Almeida, C. A. et al.

qualidade superior em relação à composição nutricional e às características físico-químicas (QIN; ZHONG, 2016). Após a extração do óleo, também é importante atentar para suas características sensoriais, como aparência, odor, sabor e cor. O azeite extraído da polpa do abacate apresenta coloração que varia do verde esmeralda límpido ao amarelado, odor característico e sabor suave ou amargo, variando de acordo com o método de extração e a variedade do fruto.

Quanto ao aquecimento, a estabilidade do azeite de abacate é semelhante à do azeite de oliva (BERASATEGI et al., 2012). A preservação das características físico-químicas do azeite de abacate após aquecimento $\left(180{ }^{\circ} \mathrm{C} \pm 5^{\circ} \mathrm{C} / 16 \mathrm{~h}\right.$ ) e armazenamento (temperatura ambiente/12 meses) pode variar entre as espécies do fruto (JORGE, 2014). O aquecimento à temperatura de $180{ }^{\circ} \mathrm{C}$ por mais de 4 horas aumenta significativamente o índice de peróxidos, dienos conjugados, $\rho$-anisidina e compostos polares, além de reduzir a estabilidade oxidativa do azeite, tornando-o impróprio para consumo (JORGE, 2014).

Em relação ao perfil nutricional, o azeite de abacate caracteriza-se por apresentar teores elevados do ácido graxo monoinsaturado oleico, teor intermediário do ácido graxo saturado palmítico e baixo teor dos ácidos graxos poli-insaturados linoleico e $\alpha$-linolênico (SOARES et al., 1992; TANGO et al., 2004). O teor de esteróis, tocoferóis e carotenoides também se apresenta como outra qualidade do azeite.

Os principais esteróis presentes são colesterol, campesterol, estigmasterol, $\beta$-sitosterol, delta-5-avenasterol, sitostanol, clerosterol e campestanol, enquanto o principal tocoferol é o $\alpha$-tocoferol (vitamina E). Dentre os carotenoides, os principais compostos são luteína, $\alpha$-caroteno, $\beta$-caroteno, zeaxantina, neoxantina e violaxantina. O perfil lipídico do azeite de abacate pode ser observado na Tabela 3.

Deve-se destacar que o perfil de ácidos graxos do azeite, bem como de esteróis, tocoferóis e carotenoides, pode variar de acordo com o método de extração (REDDY et al., 2012; JORGE, 2014), a característica do óleo (bruto ou refinado) e entre as espécies de abacate (Tabelas 4 e 5). Observa-se que a composição dos

Tabela 3. Teor médio (\%) de ácidos graxos e esteróis presentes no azeite das principais variedades de abacate.

\begin{tabular}{lr} 
& Teor Médio (\%) \\
\hline Ácidos graxos saturados & \\
ácido palmítico [16:0] & 23,5 \\
ácido esteárico [18:0] & 0,9 \\
ácido caprílico [8:0] & \\
ácido cáprico [10:0] & Traços \\
ácido láurico [12:0] & \\
ácido mirístico [14:0] & \\
Ácidos graxos insaturados & \\
ácido palmitoleico [16:1 n-7] & 7,4 \\
ácido oleico [18:1 n-9] & 52,0 \\
ácido linoleico [18:2 n-6] & 14,8 \\
ácido $\alpha$-linolênico [18:3 n-3] & 1,5 \\
Esteróis & \\
$\beta$-sitosterol & 85,3 \\
colesterol & 1,4 \\
outros & 13,3 \\
\hline Adaptado de Soas &
\end{tabular}

Adaptado de Soares e Ito (2000).

Tabela 4. Perfil de ácidos graxos do azeite de abacate das variedades Margarida, Quintal, Fortuna, Ouro Verde, Hass e Fuerte.

\begin{tabular}{|c|c|c|c|c|c|c|c|}
\hline \multicolumn{7}{|c|}{ Composição centesimal (valor em \%) } & \multirow{3}{*}{ Referência } \\
\hline \multirow{2}{*}{ Variedade } & Palmítico & Esteárico & Palmitoleico & Oleico & Linoleico & $\alpha$-linolênico & \\
\hline & {$[16: 0]$} & {$[18: 0]$} & $\overline{[16: 1 n-7]}$ & [18:1 n-9] & [18:2 n-6] & {$[18: 3 n-3]$} & \\
\hline \multirow{3}{*}{ Margarida } & 23,3 & - & 2,6 & 57,3 & 14,8 & 1,25 & (JORGE, 2014) \\
\hline & 22,7 & 1,1 & 3,9 & 55,8 & 15,3 & 1,03 & (SALGADO et al., 2008) \\
\hline & 20,3 & 0,6 & 3,4 & 51,7 & 13,8 & - & (ZÜGE, 2015) \\
\hline \multirow{2}{*}{ Quintal } & 19,8 & 0,9 & 4,2 & 66,2 & 8,8 & - & (SALGADO et al., 2008) \\
\hline & 19 & 0,5 & 7,6 & 65,2 & 9,3 & - & (TANGO et al., 2004) \\
\hline \multirow{2}{*}{ Fortuna } & 20,7 & 0,2 & 11,2 & 50,3 & 13,5 & 1,76 & (MASSAFERA et al., 2010) \\
\hline & 20,5 & 0,5 & 9,1 & 51,4 & 15,9 & 0,97 & (GALVÃO et al., 2014) \\
\hline \multirow{2}{*}{ Ouro Verde } & 18,3 & 0,5 & 6,8 & 60,6 & 13,2 & - & (TANGO et al., 2004) \\
\hline & 28,1 & - & 8,7 & 36,4 & 22,9 & 3,22 & (MASSAFERA et al., 2010) \\
\hline \multirow{4}{*}{ Hass } & 28,2 & 0,7 & 5,7 & 50,9 & 13,9 & 0,58 & (RANADE; THIAGARAJAN, 2015) \\
\hline & 19,4 & - & 11,3 & 54,7 & 13,2 & 0,83 & (JORGE, 2014) \\
\hline & 8,2 & 0,3 & 5,1 & 64,7 & 14,5 & 3,93 & (OLIVEIRA et al., 2013) \\
\hline & 24,5 & 0,3 & 13,3 & 47,7 & 14,2 & - & (TANGO et al., 2004) \\
\hline \multirow{3}{*}{ Fuerte } & 11,5 & 0,2 & 9,8 & 56,6 & 14,2 & 2,45 & (OLIVEIRA et al., 2013) \\
\hline & 20,8 & 0,7 & 5,6 & 62,9 & 9,3 & 0,37 & (SALGADO et al., 2008) \\
\hline & 20,2 & 0,4 & 7,9 & 61,4 & 10,1 & - & (TANGO et al., 2004) \\
\hline Hass + Fuerte & 22,7 & 0,4 & 12,7 & 50,5 & 12,7 & 0,67 & (FERRARI, 2015) \\
\hline
\end{tabular}


Perfil nutricional e benefícios do azeite de abacate (Persea americana): uma revisão integrativa

Nogueira-de-Almeida, C. A. et al.

Tabela 5. Teor de esteróis e tocoferóis do azeite de abacate das variedades Margarida, Hass e Fuerte.

\begin{tabular}{|c|c|c|c|c|c|c|c|c|c|c|}
\hline \multirow[b]{2}{*}{ Variedade } & \multicolumn{9}{|c|}{$(\mathrm{mg} / 100 \mathrm{~g})$} & \multirow[b]{2}{*}{ Referência } \\
\hline & 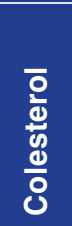 & 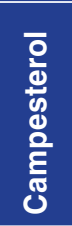 & 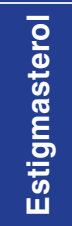 & $\begin{array}{l}\overline{0} \\
\frac{0}{9} \\
\frac{5}{8} \\
0 \\
\frac{0}{0} \\
0\end{array}$ & 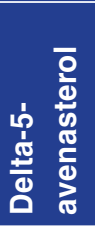 & 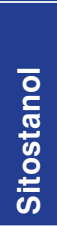 & $\begin{array}{l}\overline{0} \\
\frac{0}{5} \\
\frac{0}{0} \\
\frac{0}{0}\end{array}$ & $\begin{array}{l}\overline{\mathrm{o}} \\
\frac{\mathrm{d}}{\mathrm{d}} \\
\frac{\mathrm{o}}{\mathrm{E}} \\
\overline{\mathrm{J}}\end{array}$ & $\begin{array}{l}\overline{0} \\
\frac{5}{6} \\
8 \\
0 \\
\frac{8}{8}\end{array}$ & \\
\hline \multirow{2}{*}{ Margarida } & - & 1,8 & 1,1 & 82,3 & 1,1 & - & - & - & 7,02 & (JORGE, 2014) \\
\hline & 0,3 & 6,6 & 1,5 & 71,8 & 7,0 & 6 & 13,4 & 0,7 & 6,40 & (SALGADO et al., 2008) \\
\hline \multirow{2}{*}{ Hass } & - & 1,4 & 0,8 & 76,9 & 1,4 & - & - & - & 6,11 & (JORGE, 2014) \\
\hline & - & - & - & - & - & - & - & - & 5,36 & (VINHA et al., 2013) \\
\hline Fuerte & 1,8 & 6,3 & 0,8 & 8,7 & 1,8 & - & - & - & - & (SALGADO et al., 2008) \\
\hline
\end{tabular}

Tabela 6. Características físico-químicas do azeite de abacate das variedades Margarida, Fortuna, Hass e Fuerte.

\begin{tabular}{|c|c|c|c|c|c|c|c|c|}
\hline \multirow{3}{*}{ Variedade } & \multicolumn{7}{|c|}{ Índices (características) } & \multirow{3}{*}{ Referência } \\
\hline & 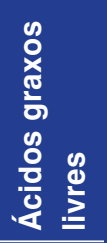 & 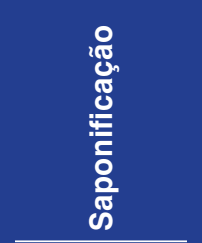 & 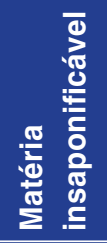 & $\begin{array}{l}\frac{0}{0} \\
\frac{0}{x} \\
\frac{0}{6} \\
0\end{array}$ & 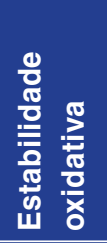 & 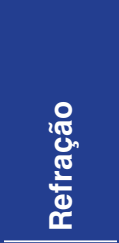 & 응 & \\
\hline & $(\%)$ & $\left(\mathrm{mg} \mathrm{KOH} \mathbf{g}^{-1}\right)$ & $(\%)$ & $\left(\operatorname{meq~} \mathrm{O}_{2} \mathrm{~kg}^{-1}\right)$ & (h) & $\left(40^{\circ} \mathrm{C}\right)$ & $(\mathrm{g} \mathrm{l} / 2 / 100 \mathrm{~g})$ & \\
\hline \multirow{2}{*}{ Margarida } & 1,82 & 194,92 & 1,19 & 4,98 & 3,87 & 1,4607 & 80,8 & (JORGE, 2014) \\
\hline & 0,36 & 181,68 & 1,60 & 9,71 & - & - & 92,9 & (SALGADO et al., 2008) \\
\hline Fortuna & - & 119,50 & - & 2,60 & - & 1,4637 & 70,4 & (GALVÃO et al., 2014) \\
\hline Hass & 0,79 & 195,79 & 0,76 & 5,54 & 5,57 & 1,4615 & 82,8 & (JORGE, 2014) \\
\hline Hass + Fuerte & 0,57 & 197,05 & 1,85 & 6,32 & 4,35 & 1,4625 & 79,0 & (FERRARI, 2015) \\
\hline
\end{tabular}

ácidos graxos constituintes do azeite bruto é similar entre as diversas variedades, não sendo possível afirmar qual apresenta maior teor de ácido oleico, linoleico ou $\alpha$-linolênico.

O teor de ácido oleico merece destaque, pois se apresenta elevado em todas as variedades. Já o baixo teor de ácidos graxos poli-insaturados é considerado bom, do ponto de vista da estabilidade do azeite, uma vez que estes, devido à quantidade de duplas ligações, são mais susceptíveis à oxidação (FERRARI, 2015). Dentre os esteróis, o principal constituinte encontrado é o $\beta$-sitosterol. Convém ressaltar que o extrato da semente do abacate pode apresentar teores mais elevados de compostos fenólicos, flavonoides e carotenoides, comparando-se ao extrato da polpa da fruta (DABAS et al., 2013; VINHA et al., 2013).

Além do perfil de ácidos graxos, esteróis e tocoferóis, as características físico-químicas também devem ser avaliadas para atestar a segurança do azeite para o consumo humano (MORETTO; FETT, 1998). Dentre elas, podem-se destacar: 1) Teor de ácidos graxos livres e índice de acidez: revelam o estado de conservação do azeite; 2) Índice de saponificação: demonstra a presença de ácidos graxos de baixo peso molecular no óleo; 3) Matéria insaponificável: inclui as substâncias que não podem ser saponificadas, correspondendo aos esteróis, tocoferóis, carotenos, entre outros; 4) Índice de peróxido: indica a rancidez oxidativa do óleo; 5) Índice de estabilidade oxidativa: indica a duração (em horas) até o início da perda da estabilidade oxidativa do óleo, decorrente das reações de oxidação dos lipídios; 6) Índice de refração: utilizado na comparação da massa molar média entre os óleos, relacionado com o grau de saturação das ligações; 7) Índice de iodo: determina o grau de insaturação de um óleo.

Estes índices servem para identificação, avaliação e determinação da qualidade da maioria dos óleos e gorduras (MORETTO; FETT, 1998). As características físico-químicas do azeite de abacate entre as diferentes variedades podem ser observadas na Tabela 6.

É possível observar que as características físico-químicas do azeite de abacate atendem, na maioria das amostras, às especificações do Codex Alimentarius (CODEX ALIMENTARIUS, 2015) para óleos virgens. Contudo, é importante salientar que tanto o Codex Alimentarius quanto a Agência Nacional de Vigilância Sanitária (ANVISA, 2005), através da Resolução RDC n. ${ }^{\circ} 270$ de 2005, que estabelece o regulamento técnico para óleos vegetais no Brasil, não fornecem ainda valores específicos e de referência para as características físico-químicas do azeite de abacate. 


\subsection{Benefícios do consumo do azeite de abacate}

O provável motivo do consumo ainda reduzido de azeite de abacate consiste, principalmente, na falta de conhecimento de seus benefícios à saúde por parte dos consumidores (OLIVEIRA, 2011).

O azeite obtido a partir do abacate possui propriedades físico-químicas semelhantes às do azeite de oliva, principalmente pelo seu perfil de esteróis e ácidos graxos, em especial o ácido oleico (SALGADO et al., 2008). $\mathrm{O}$ azeite de oliva é um produto altamente consumido no Brasil, porém de origem importada, e o azeite de abacate possui forte potencial para substituí-lo (TANGO et al., 2004; MASSAFERA et al., 2010) ou para ser utilizado conjuntamente em óleos mistos, substituindo o óleo de soja (SALGADO et al., 2008).

O perfil de ácidos graxos e os compostos bioativos presentes no azeite de abacate possuem propriedades benéficas à saúde humana. O consumo de alimentos fontes de ácidos graxos monoinsaturados, como o ácido oleico, pertencente à série ômega-9, auxilia na redução plasmática das lipoproteínas de baixa densidade (LDL-colesterol) e colesterol total, apresentando, como consequência, efeitos benéficos à saúde por meio da redução do risco de doenças cardiovasculares (VAFEIADOU et al., 2015; JORIS; MENSINK, 2016).

Outra característica do azeite de abacate é o teor de compostos fenólicos, como as procianidinas, catequinas, vanilinas, ácido hidroxifenilacético, entre outros (RUEDA et al., 2016), apesar de o conteúdo fenólico ser maior no óleo extraído da semente em relação ao extraído da polpa (WANG et al., 2010). Estes compostos estão associados à prevenção de doenças ocasionadas pela presença de radicais livres, tais como inflamação crônica, hipertensão arterial, diabetes, asma, cardiopatias e doença de Alzheimer (MANACH et al., 2004).

Destacam-se também as elevadas concentrações de tocoferóis (RUEDA et al., 2016), que atuam como nutrientes antioxidantes no plasma. Em seres humanos, os tocoferóis possuem a capacidade de prevenir danos celulares por inibir a peroxidação lipídica e a formação de radicais livres, reduzindo o risco de doenças cardiovasculares (CATANIA et al., 2009).

Já os principais carotenoides, presentes no azeite de abacate, são: luteína, $\alpha$-caroteno, $\beta$-caroteno e zeaxantina. Estudos epidemiológicos sugerem que estes carotenoides exercem papel protetor importante contra doenças cardiovasculares, oculares e alguns tipos de cânceres (TODD et al., 1999; BUIJSSE et al., 2005; KRINSKY; JOHNSON, 2005), principalmente devido à a sua atividade antioxidante (McNULTY et al., 2008), regulação do crescimento celular e modulação da expressão gênica (RAO; RAO, 2007).
Dentre os fitoesteróis contidos no azeite de abacate, destaca-se o $\beta$-sitosterol, que tem estrutura química similar à do colesterol e apresenta propriedades anti-inflamatórias e antimicrobianas (DUARTE et al., 2016), com capacidade de exercer efeitos benéficos sobre a imunidade (BOUIC, 2002), em seres humanos. Além disso, a presença de $\beta$-sitosterol na dieta permite que este se associe às demais gorduras e impeça sua absorção, sendo capaz de reduzir a absorção de colesterol no intestino delgado através de mecanismos de competição. Tal fato pode ser explicado pela semelhança entre suas estruturas químicas (RODRIGUES et al., 2004).

Em modelos animais, estudos de intervenção têm verificado que o azeite de abacate extraído do avocado é capaz de prevenir a disfunção mitocondrial cerebral e hepática induzida pelo diabetes, associado à diminuição do estresse oxidativo e da peroxidação lipídica (ORTIZ-AVILA et al., 2015a,b), além de reduzir a resistência à insulina (TORO-EQUIHUA et al., 2016). Os estudos com roedores também demonstram que a suplementação dietética com azeite de abacate contribui para a regulação do perfil lipídico e redução dos marcadores bioquímicos de risco cardiovascular, como LDL-colesterol e proteína-C-reativa (ABBOUD et al., 2015).

Apesar da excelente composição do azeite de abacate e seu potencial benefício, a quantidade de estudos que avaliam os efeitos do consumo sobre a saúde humana é escassa, abrindo-se um vasto campo para novas pesquisas. Não foram encontrados estudos do tipo ensaio clínico randomizado, meta-análises e revisões sistemáticas com seres humanos, capazes de atestar os benefícios da ingestão do referido azeite. Atualmente, as pesquisas concentram-se em avaliar os benefícios advindos do consumo da polpa do fruto e do extrato obtido da semente.

\subsection{Benefícios do consumo da polpa e do extrato da semente do abacate}

O consumo da polpa de abacate do tipo avocado, variedades Fuerte e Hass, está associado à redução do colesterol total, LDL-colesterol e triglicérides, bem como ao aumento significativo da lipoproteína de alta densidade (HDL-colesterol) em humanos, além de menores valores de peso corporal, índice de massa corporal (IMC) e circunferência da cintura (CREDIDIO, 2010; FULGONI III et al., 2013).

Os benefícios do consumo da fruta para a saúde cardiovascular são confirmados por diversos estudos (SOARES; ITO, 2000; DREHER; DAVENPORT, 2013; DUARTE et al., 2016). Os autores atribuem tais benefícios às concentrações de ácido oleico, $\beta$-sitosterol, campesterol, estigmasterol e tocoferóis presentes na fração lipídica da fruta, bem como ao teor de fibras, minerais e vitaminas lipossolúveis. 
Perfil nutricional e benefícios do azeite de abacate (Persea americana): uma revisão integrativa

Nogueira-de-Almeida, C. A. et al.

Além disso, o extrato aquoso ou etanólico obtido da semente, da folha ou da casca também pode apresentar propriedades benéficas. Estudos in vitro demonstram que esse extrato possui atividade inseticida, fungicida, antimicrobiana e antiprotozoário (ABE et al., 2005; GUZMÁN-RODRÍGUEZ et al., 2013; JIMÉNEZ-ARELLANES et al., 2013; AKINPELU et al., 2014). A baixa toxicidade e genotoxicidade do extrato da semente de abacate avaliada em estudos in vivo permite que o mesmo seja utilizado como produto farmacêutico, comestível ou cosmético (PADILLA-CAMBEROS et al., 2013).

Estudos de revisão (DABAS et al., 2013) e em modelos animais (OJEWOLE et al., 2007; BRAl et al., 2014) também demonstraram os benefícios do extrato no tratamento de condições inflamatórias, hipertensão, diabetes e hipercolesterolemia, além de propriedades hepatoprotetoras. Tais propriedades benéficas à saúde também precisam ser avaliadas após o consumo do azeite de abacate por seres humanos, em novos estudos de intervenção.

\section{Conclusão}

O azeite de abacate se destaca por seu alto teor de ácidos graxos monoinsaturados e por ser uma excelente fonte de compostos bioativos, como tocoferóis, carotenoides e fitoesteróis. Estudos de intervenção com seres humanos avaliando os efeitos do consumo do azeite sobre a saúde são escassos. Entretanto, análises em modelos animais, aliadas às análises de qualidade e segurança, atestam os benefícios e a possibilidade de introdução do referido azeite para uso comestível, substituindo óleos de qualidade inferior para a saúde humana e criando uma nova alternativa culinária ao consumidor.

\section{Referências}

ABBOUD, R. S.; PEREIRA, V. A.; COSTA, C. A. S.; BOAVENTURA, G. T.; CHAGAS, M. A. The action of avocado oil on the lipidogram of wistar rats submitted to prolonged androgenic stimulum. Nutrición Hospitalaria, v. 32, n. 2, p. 696-701, 2015. PMid:26268101.

ABE, F.; NAGAFUJI, S.; OKAWA, M.; KINJO, J.; AKAHANE, H.; OGURA, T.; MARTINEZ-ALFARO, M. A.; REYES-CHILPA, R. Trypanocidal constituents in plants 5. Evaluation of some mexican plants for their trypanocidal activity and active constituents in the seeds of Persea americana. Biological \& Pharmaceutical Bulletin, v. 28, n. 7, p. 1314-1317, 2005. http://dx.doi.org/10.1248/ bpb.28.1314. PMid:15997123.

AGÊNCIA NACIONAL DE VIGILÂNCIA SANITÁRIA - ANVISA. Resolução RDC n² 270, de 22 de setembro de 2005. Regulamento técnico para óleos vegetais, gorduras vegetais e creme vegetal. Diário Oficial [da] República Federativa do Brasil, Brasília, DF, 22 set. 2005. Disponível em: <http://portal.anvisa.gov.br/ documents/33880/2568070/RDC_270_2005.pdf/ c27660a1b6ac-4a38-aadc-956929c80b7b>. Acesso em: 17 jul. 2017.
AKINPELU, D. A.; AIYEGORO, O. A.; AKINPELU, O. F.; OKOH, A. I. Stem bark extract and fraction of Persea americana (Mill.) exhibits bactericidal activities against strains of Bacillus cereus associated with food poisoning. Molecules, v. 20, n. 1, p. 416-429, 2014. http://dx.doi.org/10.3390/molecules20010416. PMid:25558854

BERASATEGI, I.; BARRIUSO, B.; ANSORENA, D.; ASTIASARAN, I. Stability of avocado oil during heating: comparative study to olive oil. Food Chemistry, v. 132, n. 1, p. 439-446, 2012. http:// dx.doi.org/10.1016/j.foodchem.2011.11.018. PMid:26434313.

BOUIC, P. J. Sterols and sterolins: new drugs for the immune system. Drug Discovery Today, v. 7, n. 14, p. 775-778, 2002. http://dx.doi.org/10.1016/S1359-6446(02)02343-7. PMid:12547034.

BRAI, B. I.; ADISA, R. A.; ODETOLA, A. A. Hepatoprotective properties of aqueous leaf extract of Persea americana, Mill (Lauraceae) 'Avocado' against $\mathrm{CCl}_{4}$-induced damage in rats. African Journal of Traditional, Complementary, and Alternative Medicines, v. 11, n. 2, p. 237-244, 2014. http://dx.doi.org/10.4314/ ajtcam.v11i2.2. PMid:25435602.

BUIJSSE, B.; FESKENS, E. J.; SCHLETTWEIN-GSELL, D.; FERRY, M.; KOK, F. J.; KROMHOUT, D.; GROOT, L. C. Plasma carotene and alpha-tocopherol in relation to 10-y all-cause and causespecific mortality in European elderly: the survey in europe on nutrition and the elderly, a concerted action seneca. The American Journal of Clinical Nutrition, v. 82, n. 4, p. 879-886, 2005. http://dx.doi.org/10.1093/ajcn/82.4.879. PMid:16210720.

CAMPOS, J. S. Cultura racional do abacateiro. São Paulo: Icone, 1985. $150 \mathrm{p}$.

CAMPOS, R. A. Otimização de método de extração de óleo presente em polpa de abacate. 2009. 70 f. Dissertação (Mestrado em Química)--Instituto de Química, Universidade Estadual Paulista, Araraquara, 2009.

CATANIA, A. S.; BARROS, C. R.; FERREIRA, S. R. G. Vitaminas e minerais com propriedades antioxidantes e risco cardiometabólico: controvérsias e perspectivas. Arquivos Brasileiros de Endocrinologia \& Metabologia, v. 53, n. 5, p. 550-559, 2009. http:// dx.doi.org/10.1590/S0004-27302009000500008. PMid: 19768246.

CODEX ALIMENTARIUS. Codex standard for named vegetable oils (CODEX-STAN 210 - 1999) - Adapted in 1999 - revision: 2001, 2003, 2009 - amendment: 2005, 2011, 2013 and 2015. Rome: Food and Agriculture Organization of the United Nations; World Health Organization, 2015. 13 p.

CREDIDIO, E. V. Estudo do efeito do abacate nos lipídeos sanguíneos em humanos. 2010. 165 f. Tese (Doutorado em Ciência de Alimentos)--Faculdade de Engenharia de Alimentos, Universidade Estadual de Campinas, Campinas, 2010.

DABAS, D.; SHEGOG, R. M.; ZIEGLER, G. R.; LAMBERT, J. D. Avocado (Persea americana) seed as a source of bioactive phytochemicals. Current Pharmaceutical Design, v. 19, n. 34 , p. 6133-6140, 2013. PMid:23448442. 
Perfil nutricional e benefícios do azeite de abacate (Persea americana): uma revisão integrativa Nogueira-de-Almeida, C. A. et al.

DAIUTO, É. R.; VIEITES, R. L.; TREMOCOLDI, M. A.; VILEIGAS, D. F. Estabilidade físico-química de um produto de abacate acondicionado em diferentes embalagens e conservado pelo frio. Alimentos e Nutrição, v. 21, n. 1, p. 97-105, 2010.

DREHER, M. L.; DAVENPORT, A. J. Hass avocado composition and potential health effects. Critical Reviews in Food Science and Nutrition, v. 53, n. 7, p. 738-750, 2013. http://dx.doi.org/1 0.1080/10408398.2011.556759. PMid:23638933.

DUARTE, P. F.; CHAVES, M. A.; BORGES, C. D.; MENDONÇA, C. R. B. Avocado: characteristics, health benefits and uses. Ciência Rural, v. 46, n. 4, p. 747-754, 2016. http://dx.doi. org/10.1590/0103-8478cr20141516.

FERRARI, R. A. Nota científica: caracterização físico-química do óleo de abacate extraído por centrifugação e dos subprodutos do processamento. Brazilian Journal of Food Technology, v. 18, n. 1, p. 79-84, 2015. http://dx.doi.org/10.1590/1981-6723.4014.

FRANCISCO, V. L. F. S.; BAPTISTELLA, C. S. L. Cultura do abacate no Estado de São Paulo. Informações Econômicas, v. 35, n. 5 , p. $27-41,2005$

FULGONI III, V. L.; DREHER, M.; DAVENPORT, A. J. Avocado consumption is associated with better diet quality and nutrient intake, and lower metabolic syndrome risk in US adults: results from the National Health and Nutrition Examination Survey (NHANES) 2001-2008. Nutrition Journal, v. 12, n. 1, p. 1-6, 2013. http://dx. doi.org/10.1186/1475-2891-12-1. PMid:23282226.

GALVÃO, M. S.; NARAIN, N.; NIGAM, N. Influence of different cultivars on oil quality and chemical characteristics of avocado fruit. Food Science and Technology, v. 34, n. 3, p. 539-546, 2014. http://dx.doi.org/10.1590/1678-457x.6388.

GÓMEZ-LÓPEZ, V. M. Characterization of Avocado (Persea americana Mill.) varieties of low oil content. Journal of Agricultural and Food Chemistry, v. 47, n. 7, p. 2707-2710, 1999. http:// dx.doi.org/10.1021/jf981206a. PMid:10552549.

GOUVEIA, H. L.; SCHAUN, J. S.; MASKE, A.; SCHEIK, L. K.; BORGES, C. D.; MENDONÇA, C. R. B. Abacate da variedade breda: características físico-químicas e teor de lipídios. In: SIMPÓSIO DE SEGURANÇA ALIMENTAR: ALIMENTAÇÃO E SAÚDE, 5., 2015, Bento Gonçalves. Anais..., Porto Alegre: Sociedade Brasileira de Ciência e Tecnologia de Alimentos - SBCTA-RS, 2015. Disponível em: <http://www.ufrgs.br/sbctarseventos/gerenciador/painel/trabalhosversaofinal/SAL169.pdf>. Acesso em: 17 jul. 2017.

GUZMÁN-RODRÍGUEZ, J. J.; LOPEZ-GOMEZ, R.; SUAREZRODRIGUEZ, L. M.; SALGADO-GARCIGLIA, R.; RODRIGUEZ-ZAPATA, L. C.; OCHOA-ZARZOSA, A.; LOPEZ-MEZA, J. E. Antibacterial activity of defensin padef from Avocado fruit (Persea Americana Var. Drymifolia) expressed in endothelial cells against Escherichia coli and Staphylococcus aureus. BioMed Research International, v. 2013, p. 986273, 2013. http://dx.doi.org/10.1155/2013/986273. PMid:24319695.
HORTIESCOLHA. Hortipedia - Abacate. Piracicaba: Departamento de Agroindústria, Alimentos e Nutrição, Escola Superior de Agricultura "Luiz de Queiroz", Universidade de São Paulo; São Paulo: Centro de Qualidade em Horticultura da Companhia de Entrepostos e Armazéns Gerais do Estado de São Paulo, 2017. Disponível em: <http://www.hortiescolha.com.br/hortipedia/ produto/abacate>. Acesso em: 7 maio 2017.

INSTITUTO BRASILEIRO DE GEOGRAFIA E ESTATÍSTICA - IBGE. Pesquisa de orçamentos familiares 2008-2009: tabelas de composição nutricional dos alimentos consumidos no Brasil. Rio de Janeiro: IBGE, 2011. Disponível em: <http://biblioteca. ibge.gov.br/visualizacao/livros/liv50002.pdf>. Acesso em: 17 jul. 2017

JIMÉNEZ-ARELLANES, A.; LUNA-HERRERA, J.; RUIZ-NICOLAS, R.; CORNEJO-GARRIDO, J.; TAPIA, A.; YEPEZ-MULIA, L. Antiprotozoal and antimycobacterial activities of Persea Americana seeds. BMC Complementary and Alternative Medicine, v. 13, n. 1, p. 109-114, 2013. http://dx.doi.org/10.1186/1472-6882-13109. PMid:23680126.

JORGE, T. S. Avaliação reológica do óleo de abacate (Persea americana mill) e estudo da estabilidade sob condições de aquecimento e armazenamento à temperatura ambiente. 2014. 82 f. Dissertação (Mestrado em Engenharia e Ciência de Alimentos)--Instituto de Biociências, Letras e Ciências Exatas, Universidade Estadual Paulista, São José do Rio Preto, 2014.

JORIS, P. J.; MENSINK, R. P. Role of cis-monounsaturated fatty acids in the prevention of coronary heart disease. Current Atherosclerosis Reports, v. 18, n. 7, p. 38-45, 2016. http:// dx.doi.org/10.1007/s11883-016-0597-y. PMid:27221500.

KOLLER, O. C. Abacaticultura. Porto Alegre: UFRGS, 1992. 138 p.

KRINSKY, N. I.; JOHNSON, E. J. Carotenoid actions and their relation to health and disease. Molecular Aspects of Medicine, v. 26, n. 6, p. 459-516, 2005. http://dx.doi.org/10.1016/j. mam.2005.10.001. PMid:16309738.

MANACH, C.; SCALBERT, A.; MORAND, C.; RÉMÉSY, C.; JIMÉNEZ, L. Polyphenols: food sources and bioavailability. The American Journal of Clinical Nutrition, v. 79, n. 5, p. 727-747, 2004. http://dx.doi.org/10.1093/ajcn/79.5.727. PMid:15113710.

MASSAFERA, G.; BRAGA COSTA, T. M.; OLIVEIRA, J. E. Composição de ácidos graxos do óleo do mesocarpo e da semente de cultivares de abacate (Persea americana, Mill.) da região de Ribeirão Preto, SP. Alimentos e Nutrição, v. 21, n. 2, p. 325-331, 2010.

MCNULTY, H.; JACOB, R. F.; MASON, R. P. Biologic activity of carotenoids related to distinct membrane physicochemical interactions. The American Journal of Cardiology, v. 101, n. 10A, p. 20D-29D, 2008. http://dx.doi.org/10.1016/j.amjcard.2008.02.004. PMid:18474269

MENDES, K. D. S.; SILVEIRA, R. C. C. P.; GALVÃO, C. M. Revisão integrativa: método de pesquisa para a incorporação de evidências 
Perfil nutricional e benefícios do azeite de abacate (Persea americana): uma revisão integrativa Nogueira-de-Almeida, C. A. et al.

na saúde e na enfermagem. Texto \& Contexto Enfermagem, v. 17, n. 4, p. 758-764, 2008. http://dx.doi.org/10.1590/S010407072008000400018.

MONTANO, G. H.; LUH, B. S.; SMITH, L. M. Extracting and refining avocado oil. Food Technology, v. 16, n. 2, p. 96-101, 1962

MORETTO, E.; FETT, R. Tecnologia de óleos e gorduras vegetais na indústria de alimentos. São Paulo: Livraria Varela, 1998. 144 p.

NÚCLEO DE ESTUDOS E PESQUISA EM ALIMENTAÇÃO - NEPA. Tabela brasileira de composição de alimentos -TACO. 4. ed. Campinas: UNICAMP, 2011. Disponível em: <http://www.cfn.org. br/wp-content/uploads/2017/03/taco_4_edicao_ampliada_e_ revisada.pdf >. Acesso em: 7 mai. 2017.

OJEWOLE, J. A.; KAMADYAAPA, D. R.; GONDWE, M. M.; MOODLEY, K.; MUSABAYANE, C. T. Cardiovascular effects of Persea americana Mill (Lauraceae) (avocado) aqueous leaf extract in experimental animals. Cardiovascular Journal of Africa, v. 18, n. 2, p. 69-76, 2007. PMid:17497042.

OLIVEIRA, M. C. Abacateiro e Oliveira como fontes de matéria-prima visando à extração do óleo. 2011. 84 f. Tese (Doutorado em Agronomia/Fitotecnia)--Universidade Federal de Lavras, Lavras, 2011.

OLIVEIRA, M. C.; PIO, R.; RAMOS, J. D.; LIMA, L. C. O.; PASQUAL, M.; SANTOS, V. A. Fenologia e características físico-químicas de frutos de abacateiros visando à extração de óleo. Ciência Rural, v. 43, n. 3, p. 411-418, 2013. http://dx.doi.org/10.1590/ S0103-84782013000300006.

ORTIZ-AVILA, O.; ESQUIVEL-MARTINEZ, M.; OLMOS-ORIZABA, B. E.; SAAVEDRA-MOLINA, A.; RODRIGUEZ-OROZCO, A. R.; CORTES-ROJO, C. Avocado oil improves mitochondrial function and decreases oxidative stress in brain of diabetic rats. Journal of Diabetes Research, v. 2015, p. 485759, 2015a. http://dx. doi. org/10.1155/2015/485759. PMid:26180820.

ORTIZ-AVILA, O.; GALLEGOS-CORONA, M. A.; SANCHEZBRIONES, L. A.; CALDERON-CORTES, E.; MONTOYA-PEREZ, R.; RODRIGUEZ-OROZCO, A. R.; CAMPOS-GARCIA, J.; SAAVEDRA-MOLINA, A.; MEJIA-ZEPEDA, R.; CORTES-ROJO, C. Protective effects of dietary avocado oil on impaired electron transport chain function and exacerbated oxidative stress in liver mitochondria from diabetic rats. Journal of Bioenergetics and Biomembranes, v. 47, n. 4, p. 337-353, 2015b. http://dx.doi. org/10.1007/s10863-015-9614-z. PMid:26060181.

PADILLA-CAMBEROS, E.; MARTINEZ-VELAZQUEZ, M.; FLORESFERNANDEZ, J. M.; VILLANUEVA-RODRIGUEZ, S. Acute toxicity and genotoxic activity of avocado seed extract (Persea americana Mill., C.v. Hass). The Scientific World Journal, v. 2013, p. 245828, 2013. http://dx.doi.org/10.1155/2013/245828. PMid:24298206.

PEREIRA, P. A. Evolução da produção mundial e nacional de abacate. 2015. 55 f. Trabalho de Conclusão de Curso (Graduação em Agronomia)--Faculdade de Agronomia e Medicina Veterinária, Universidade de Brasília, Brasília, 2015.

QIN, X.; ZHONG, J. A review of extraction techniques for avocado oil. Journal of Oleo Science, v. 65, n. 11, p. 881-888, 2016. http://dx.doi.org/10.5650/jos.ess16063. PMid:27725362.

RANADE, S. S.; THIAGARAJAN, P. A review on Persea americana Mill. (avocado) - its fruits and oil. International Journal of Pharm Tech Research, v. 8, n. 6, p. 72-77, 2015.

RAO, A.; RAO, L. Carotenoids and human health. Pharmacological Research, v. 55, n. 3, p. 207-216, 2007. http://dx.doi.org/10.1016/j. phrs.2007.01.012. PMid:17349800.

REDDY, M.; MOODLEY, R.; JONNALAGADDA, S. B. Fatty acid profile and elemental content of avocado (Persea americana Mill.) oil--effect of extraction methods. Journal of Environmental Science and Health. Part. B, Pesticides, Food Contaminants, and Agricultural Wastes, v. 47, n. 6, p. 529-537, 2012. http:// dx.doi.org/10.1080/03601234.2012.665669. PMid:22494376.

RODRIGUES, J. N.; MANCINI FILHO, J.; TORRES, R. P.; GIOIELLI, L. A. Physico-chemical characterization of a phytosterol ester enriched margarine. Revista Brasileira de Ciências Farmacêuticas, v. 40, n. 4, p. 505-520, 2004. http://dx.doi.org/10.1590/S151693322004000400008.

RUEDA, A.; SAMANIEGO-SÁNCHEZ, C.; OLALLA, M.; GIMÉNEZ, R.; CABRERA-VIQUE, C.; SEIQUER, I.; LARA, L. Combination of analytical and chemometric methods as a useful tool for the characterization of extra virgin argan oil and other edible virgin oils. role of polyphenols and tocopherols. Journal of AOAC International, v. 99, n. 2, p. 489-494, 2016. http://dx.doi. org/10.5740/jaoacint.15-0121. PMid:26953066.

SALGADO, J. M.; DANIELI, F.; REGITANO-D'ARCE, M. A. B.; FRIAS, A.; MANSI, D. N. O óleo de abacate (Persea americana Mill) como matéria-prima para a indústria alimentícia. Food Science and Technology, v. 28, n. 1, p. 20-26, 2008. http:// dx.doi.org/10.1590/S0101-20612008000500004.

SANTOS, M. A. Z.; ALICIEO, T. V. R.; PEREIRA, C. M. P.; RAMISRAMOS, G.; MENDONÇA, C. R. B. Profile of bioactive compounds in avocado pulp oil: influence of the drying processes and extraction methods. Journal of the American Oil Chemists' Society, v. 91, n. 1, p. 19-27, 2014. http://dx.doi.org/10.1007/ s11746-013-2289-X.

SOARES, H. F.; ITO, M. K. The monounsaturated fatty acid from avocado in the control of dyslipidaemia. Revista Ciencia Médica, v. 9, n. 2 , p. $47-51,2000$.

SOARES, S. E.; MANCINI FILHO, J.; DELLA MODESTA, R. C. Sensory detection limits of avocado oil in mixtures with olive oil. Revista Española de Ciencia y Tecnología de Alimentos, v. 32, n. 5, p. 509-516, 1992.

TANGO, J. S.; CARVALHO, C. R. L.; SOARES, N. B. Caracterização física e química de frutos de abacate visando a seu potencial para extração de óleo. Revista Brasileira de Fruticultura, 
Perfil nutricional e benefícios do azeite de abacate (Persea americana): uma revisão integrativa

Nogueira-de-Almeida, C. A. et al.

v. 26 , n. 1, p. 17-23, 2004. http://dx.doi.org/10.1590/S010029452004000100007

TANGO, J. S.; TURATTI, J. M. Óleo de abacate. In: Instituto de Tecnologia de Alimentos - ITAL (Ed.). Abacate: cultura, matériaprima, processamento e aspectos econômicos. Campinas: ITAL, 1992. cap. 4, p. 156-192.

TODD, S.; WOODWARD, M.; TUNSTALL-PEDOE, H.; BOLTON$\mathrm{SMITH}, \mathrm{C}$. Dietary antioxidant vitamins and fiber in the etiology of cardiovascular disease and all-causes mortality: results from the scottish heart health study. American Journal of Epidemiology, v. 150, n. 10 , p. 1073-1080, 1999. http://dx.doi.org/10.1093/ oxfordjournals.aje.a009931. PMid:10568622.

TORO-EQUIHUA, M.; VELASCO-RODRÍGUEZ, R.; LÓPEZASCENCIO, R.; VÁSQUEZ, C. Effect of an avocado oil-enhanced diet (Persea americana) on sucrose-induced insulin resistance in Wistar rats. Journal of Food and Drug Analysis, v. 24, n. 2 , p. 350-357, 2016. http://dx.doi.org/10.1016/j.jfda.2015.11.005. PMid:28911589.

TURATTI, J. M.; SANTOS, L. C.; TANGO, J. S.; ARIMA, H. K. Caracterização do óleo de abacate obtido por diferentes processos de extração. Boletim do ITAL, v. 22, p. 267-284, 1985.
VAFEIADOU, K.; WEECH, M.; ALTOWAIJRI, H.; TODD, S.; YAQOOB, P.; JACKSON, K. G.; LOVEGROVE, J. A. Replacement of saturated with unsaturated fats had no impact on vascular function but beneficial effects on lipid biomarkers, E-selectin, and blood pressure: results from the randomized, controlled dietary intervention and vascular function (DIVAS) study. The American Journal of Clinical Nutrition, v. 102, n. 1, p. 40-48, 2015. http://dx.doi.org/10.3945/ajcn.114.097089. PMid:26016869. VINHA, A. F.; MOREIRA, J.; BARREIRA, S. V. P. Physicochemical parameters, phytochemical composition and antioxidant activity of the algarvian avocado (Persea americana Mill.). The Journal of Agricultural Science, v. 5, n. 12, p. 100-109, 2013.

WANG, W.; BOSTIC, T. R.; GU, L. Antioxidant capacities, procyanidins and pigments in avocados of different strains and cultivars. Food Chemistry, v. 122, n. 4, p. 1193-1198, 2010. http://dx.doi.org/10.1016/j.foodchem.2010.03.114.

ZÜGE, L. C. B. Extração e caracterização da polpa e do óleo de abacate (Persea americana) visando a obtenção de fosfolipídeos para uso em emulsões. 2015. 116 f. Tese (Doutorado em Engenharia de Alimentos)--Universidade Federal do Paraná, Curitiba, 2015. 